\section{P3.63 THE PREVALENCE AND INCIDENCE OF ACTIVE SYPHILIS IN WOMEN IN MOROCCO, 1995-2016: MODEL-BASED ESTIMATION AND IMPLICATIONS FOR STI SURVEILLANCE}

${ }^{1}$ Eline Korenromp, ${ }^{2}$ Melanie Taylor, ${ }^{3}$ Aziza Bennani, ${ }^{4}$ Amina El-Kettani, ${ }^{5}$ Houssine El-Rhilani,

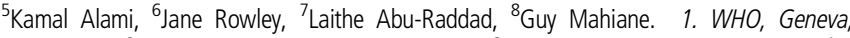
Switzerland; ${ }^{2}$ Avenir Health, Geneva, Switzerland; ${ }^{3}$ Moh Morocco, Rabat, Morocco; ${ }^{4}$ Sti Laboratory National Institute of Hygiene, Rabat, Morocco; ${ }^{5}$ Unaids Morocco, Rabat, Morocco; ${ }^{6}$ London School of Trop Med andHygiene, London, UK; ${ }^{7}$ Weill Cornell Medical College, Doha, Qatar; 8. Avenir Health, Glastonbury, CT, USA

\subsection{6/sextrans-2017-053264.298}

Introduction In 2016, the World Health Assembly adopted the global strategy 2016-2021 for STI control through the health sector, with as impact target to reduce syphilis incidence by $90 \%$ from 2018 to 2030. We applied the Spectrum STI estimation tool to estimate national adult prevalence and incidence of active syphilis in Morocco currently and over 19952015, to inform its national HIV/STI strategy, target setting and program evaluation.

Methods Syphilis prevalence levels and trends were fitted through logistic regression to data from surveys in antenatal clinics conducted during 1996-2012, and among women attending family planning clinics. Prevalence data were adjusted for diagnostic test performance. Incidence was inferred from prevalence by adjusting for the average duration of infection with active syphilis. Rates estimated for ANC women were assumed to apply to the overall female adult (15-49 years) population.

Results In 2016, syphilis prevalence was estimated to be $0.56 \%$ (95\% confidence interval, CI: $0.3 \%-1.0 \%)$. Around $21,675(10,612-37,198)$ new syphilis infections were estimated to have occurred in adult women in 2016. This is a decline from the prevalence estimate for 2000 of $1.38 \% \quad(0.87 \%-$ $2.1 \%)$.

Conclusion Periodic population-based surveys allowed Morocco to estimate syphilis prevalence and incidence trends. The latest survey was done in 2012 and so the trends are relatively uncertain after 2012, when no new surveys were conducted. Planned recording of results from routine antenatal programmatic screening, being rolled out from 2017, should soon allow updating and re-calibration of future estimations.

\section{P3.64 IS THE INCIDENCE OF NEONATAL HERPES INFECTION ON THE RISE IN THE NETHERLANDS?}

${ }^{1}$ Eline Op De Coul, ${ }^{2}$ Manisha Biekram, ${ }^{2}$ Louise Van Oeffelen, ${ }^{3}$ Jashvant Poeran, ${ }^{4}$ Wim Van Der Meijden. 'Centre For Infectious Disease Control, Rivm, Bilthoven, The Netherlands; ${ }^{2}$ Centrffor Infectious Disease Control, Rivm, Bilthoven, The Netherlands; ${ }^{3}$ Department of Population Health Science and Policy, ICAHN School of Medicine at Mount Sinai, New York; ${ }^{4}$ Beatrix Hospital, Gorinchem, The Netherlands

\subsection{6/sextrans-2017-053264.299}

Introduction Neonatal herpes can result from intrauterine, perinatal, and postnatal herpes simplex virus (HSV) transmission. Although rare, it can lead to severe disease and neonatal death. Between 1981 and 2011, neonatal herpes incidence has been monitored every five years in the Netherlands showing an increase over time. We investigated the further course of neonatal herpes incidence between 2012 and 2015, and studied longitudinal trends between 1999 and 2015. Additionally, we explored guideline adherence by health care professionals as this may contribute to changes in neonatal herpes incidence.

Methods Questionnaires were sent to all paediatric $(n=93)$, gynaecology $(n=93)$ and microbiology $(n=47)$ departments of all hospitals in the Netherlands, inquiring about the number of neonatal herpes cases and guideline adherence between 2012 and 2015. Guideline adherence from 2006-2011 was obtained from previous studies.

Incidence of neonatal HSV infections was calculated by combining data from paediatric and microbiology departments taking non-response rates into account. For trend analyses, the annual numbers of neonatal herpes cases from 1999 onwards were obtained from previous surveys and from The Netherlands Perinatal Registry (Perined). Incidence trend lines were smoothed by calculating Poisson regression splines.

Results During 2012-2015, neonatal herpes incidence was 4.8 per 100000 newborns based on survey data and 3.4 per 100000 newborns based on Perined data. Longitudinal trend analyses displayed a minor increasing trend between 1999 and 2015. Adherence of gynaecologists and paediatricians to Dutch guidelines in case of a primary maternal HSV infection was somewhat lower in 2012-2015 compared to 2006-2011 in terms of antiviral therapy provision and performance of an elective Caesarean section.

Conclusion Longitudinal analyses showed a minor increasing trend in neonatal herpes incidence. Underlying factors of this increase might be related to a lower guideline adherence by health care professionals or other factors such as an increased age of first HSV-1 infection. Efforts should be made to underline the importance of guideline adherence among gynaecologists and paediatricians.

\section{P3.65 THE ENHANCED GONOCOCCAL ANTIMICROBIAL SURVEILLANCE PROGRAM (EGASP) IN THAILAND, 2015- 2016}

${ }^{1}$ Emily Weston, ${ }^{2}$ Malai Siritrapanan, ${ }^{3}$ Sirirat Lertpruek, ${ }^{2}$ Nisit Kongkregkeat, ${ }^{3}$ Jaray Tongtoyai, ${ }^{3}$ Thitima Cherdtrakulkiat, ${ }^{3}$ Wichuda Sukwicha, ${ }^{4}$ Chatnapa Duangdee, ${ }^{5}$ Teodora Wi, ${ }^{1}$ John Papp, ${ }^{6}$ Eileen Dunne, ${ }^{7}$ Pachara Sirivongrangsan. ${ }^{1}$ Division of STD Prevention, US Centres for Disease Control and Prevention, Atlanta, USA; ${ }^{2}$ Bangrak STIS Bureau of AIDS/TB/STIS, Dept of Disease Control, Thailand Ministry of Public Health, Bangkok, Thailand; ${ }^{3}$ HIVISTD Research Program, Thailand Ministry of Health, US Centres for Disease Conotrol Collaboration, Bangkok, Thailand; ${ }^{4}$ Hospital for Tropical Diseases, Mahidol University, Bangkok, Thailand; ${ }^{5}$ Department of Reproductive Health, World Health Organisation, Geneva, Switzerland; ${ }^{6}$ Division of HIVIAIDS Prevention, US Centres for Disease Control and Prevention, Atlanta, USA; ${ }^{7}$ Department of Disease Control and Prevention, Thailand Ministry of Public Health, Bangkok, Thailand

\subsection{6/sextrans-2017-053264.300}

Introduction Antimicrobial resistant Neisseria gonorrhoea (NG) is a global public health threat, and it is critical to monitor patterns of resistance and risk factors. The Thailand Ministry of Public Health, the Centres for Disease Control and Prevention and World Health Organisation began the Enhanced Gonococcal Antimicrobial Surveillance Programme (EGASP) in November 2015. Thailand is the first EGASP country to systematically monitor trends in NG antimicrobial susceptibilities. Methods Surveillance occurs in 2 sites: Bangrak Hospital (BH) and Silom Community Clinic at Tropical Medicine (SCC). Men receiving HIV voluntary counselling and testing were routinely asked about urethral symptoms. Symptomatic men had demographic and clinical data collected and a urethral swab for Gram stain and NG culture. All positive cultures 